\title{
AN ANTENNA SOLUTION FOR MIMO CHANNELS: THE SWITCHED PARASITIC ANTENNA
}

Mattias Wennström

\author{
Signals and Systems Group \\ Uppsala University \\ mw@signal.uu.se
}

Technical area: Antenna and RF subsystems

\begin{abstract}
We investigate the switched parasitic antenna (SPA), which is a novel technique for electronically directing the radiation pattern, in a MIMO system. The correlation between the received signal modes are shown to be sufficiently low to yield a diversity gain. The capacity limit using the SPA is investigated for different SPA configurations and it is found that the capacity is comparable with an array antenna configuration in certain situations. Finally, a space time block coding scheme is used to evaluate the bit error rate of a MIMO-SPA system. It is found that the SPA requires a $5 \mathrm{~dB}$ higher SNR than an antenna array solution to achieve a $\mathrm{BER}=10^{-2}$. However, the array antenna requires a radio transceiver for every antenna, as opposed to the SPA which uses only one transceiver.
\end{abstract}

\section{INTRODUCTION}

Recent information theory results have demonstrated an enormous capacity potential of wireless systems with multiple antennas at both transmitter and receiver, so called multiple input, multiple output (MIMO) systems [1]. An unfortunate aspect of MIMO systems is the high cost of multiple radio transceivers at the access point and at the user terminal. Furthermore, it is expensive to calibrate and maintain antenna arrays with many antenna elements. Recently, switched parasitic antennas (SPAs) have been subject of an increased interest in the literature, for improving capacity in indoor LANs [2], as a diversity antenna [3, 4] and for tracking of base-stations [5].

In a sense, SPA offers characteristics similar to an array antenna with several fixed beams, but is more compact in size, and might be more suitable on certain mobile equipments. This paper will examine the use of SPAs in MIMO systems, where the SPA is primarily used at the user equipment (UE), in a realistic flat fading environment. It has been observed that when the fades of the MIMO receive channels are correlated, the channel capacity can be significantly smaller than when the fades are independent and identically distributed (i.i.d.) [6]. Hence, we study the capacity of the SPA and compare this capacity with a completely uncorrelated scenario. Furthermore,

\footnotetext{
This work was supported by NUTEK.

This work was supported in part by the Swedish Foundation for Strategic Research, under the Personal Computing and Communications Program.
}

Thomas Svantesson

\author{
Department of Signals and Systems \\ Chalmers University of Technology \\ tomaso@s2.chalmers.se
}

we apply space time block coding (STBC) techniques to examine how this correlation affects the BER of a SPA system.

\section{SWITCHED PARASITIC ANTENNAS}

SPAs offering directional patterns goes back to the early work of Yagi and Uda in the 1930's. For mobile communications, Vaughan [4] gave some examples of this technology to provide angle diversity as two parasitics on a mobile phone handset. The concept is to use a single active antenna element, connected to a radio transceiver, in a structure with one or several passive antenna elements, operating near resonance. The passive elements are called parasitic elements (PE) and act together with the active element to form an array, as in the well known Yagi-Uda array [7]. To alter the radiation pattern, the termination impedances of the PEs are switchable, to change the current flowing in those elements. The PE become reflectors when shorted to the ground plane using pin diodes [8] and when not shorted, the PE have little effect on the antenna characteristics. The receiver is always connected to the center antenna element so there are no switches in the RF direct signal path.

The parasitic antennas can be designed using monopoles on a ground plane [4],[8] or as parasitic patch antennas [9]. In this paper we consider the monopole on the ground plane for its omnidirectional properties. Examples of parasitic antennas are shown in Figure 1 for a 4-direction symmetry and in Figure 2 for a 3-direction symmetric antenna. The antenna in Figure 2 have an additional circle of parasitic elements that always are shorted to ground. The effect of this arrangement is an increased directivity as their length are shorter than the corresponding resonant length $(\approx \lambda / 4)$ and will lead the induced emf [7].

The lengths and distances displayed in Figure 1 and 2 are not optimal in any way. The lengths can be adjusted to give the antenna certain characteristics, such as directivity and/or dual band tuning, as demonstrated in [8], where a genetic algorithm approach was taken to optimize a six element switched beam antenna. If the parasitics are moved closer to the active element, the mutual coupling increases and the change in the radiation pattern when switching is greater, however, the antenna impedance changes also more dramatically, which makes the antenna matching difficult. Hence, a too large mutual coupling renders in an inefficient antenna. The trade-off is thereby between compactness and high directivity on one 


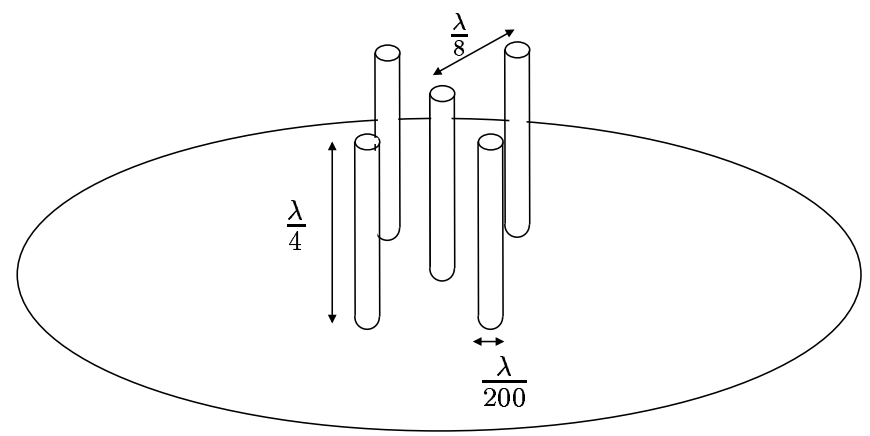

Figure 1: A five element monopole SPA. The center element is active and connected to the transceiver. The four passive antenna elements can be switched in or out of resonance using appropriately biased pin diodes.

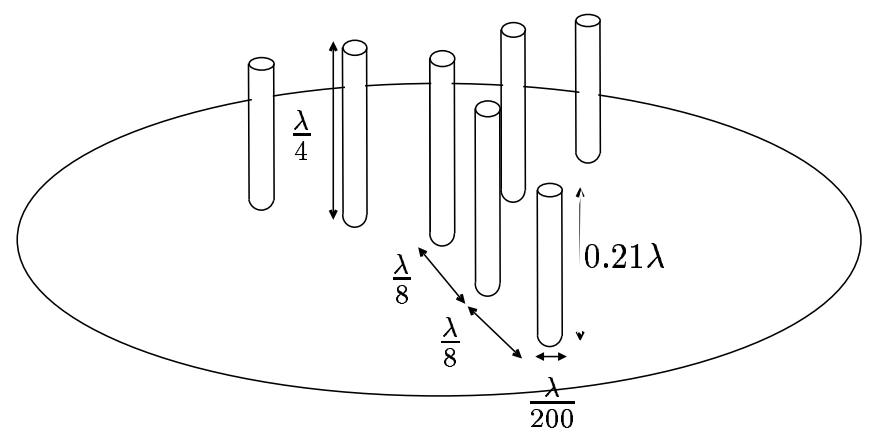

Figure 2: A seven element monopole SPA. The center element is active and connected to the transceiver. The three passive antenna elements closest to the active can be switched in or out of resonance using appropriately biased pin diodes. The three outermost monopoles are hardwired to ground.

hand and antenna efficiency on the other.

The antennas in Figures 1 and 2 was simulated using HFSS (High Frequency Structure Simulator) from Agilent Technologies Inc. which is a $3 \mathrm{D}$ simulator using the finite element method to solve for the electromagnetic field. The software was used to calculate the far-field radiation pattern of the antenna for different settings of the switched parasitics. The monopole elements were cylindrical with an length to radius ratio $l / r=100$ which have a first resonance at approximately $0.24 \lambda$ [7]. The monopoles were assumed to be perfect conductors which forces the electrical field to be normal to the surface. Furthermore, to shorten the simulation time, the ground plane was assumed to be of infinite extent. The chosen frequency was $2.15 \mathrm{GHz}$, suitable for the downlink in UMTS FDD mode. Hence, the wavelength is $\lambda=0.1395$ meter.

The far-field power radiation pattern for three shorted parasitics and one open for parasitic antenna 1 is shown in Figure 3 and the corresponding plot for Figure 2 is shown in Figure 4 for two shorted and one open parasitic. The directivity of the two antennas are $9.9 \mathrm{~dB}$ and $10.0 \mathrm{~dB}$ respectively.

\section{MIMO CHANNEL CAPACITY AND DIVERSITY GAIN}

To achieve a high capacity in MIMO systems or a large diversity gain, the signals received by different settings of the parasitics, called the $M$ modes, must have low correlation (ideally zero). Hence, we define the correlation coefficient of the signal voltages received by two patterns as [10]

$$
\rho_{12}=\int_{0}^{2 \pi} \int_{0}^{\pi} S(\theta, \phi) E_{1}(\theta, \phi) \cdot E_{2}^{*}(\theta, \phi) \sin \theta d \theta d \phi
$$

where the two far field patterns $E_{1}(\theta, \phi)$ and $E_{2}(\theta, \phi)$ are normalized as

$$
\int_{0}^{2 \pi} \int_{0}^{\pi} S(\theta, \phi)\left|E_{i}(\theta, \phi)\right|^{2} \sin \theta d \theta d \phi=1
$$

for $i=1,2$. Above, $S(\theta, \phi)$ is the pdf of the incident waves.

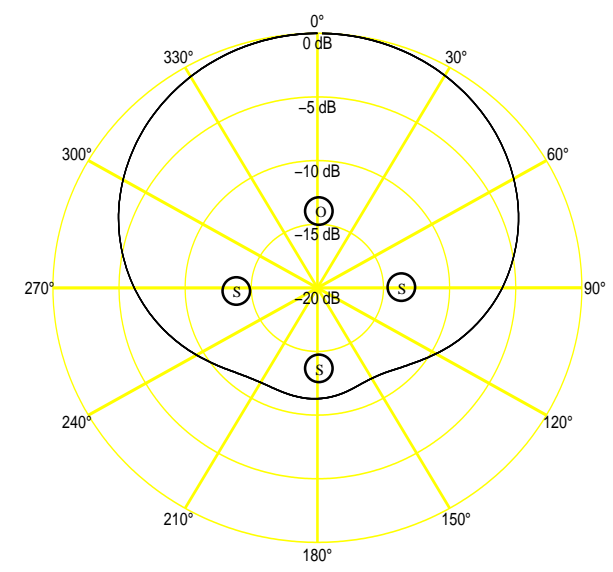

Figure 3: Power radiation pattern of the five element monopole antenna shown in Figure 1 with three parasitics shorted $(S)$ to ground and one open $(O)$.

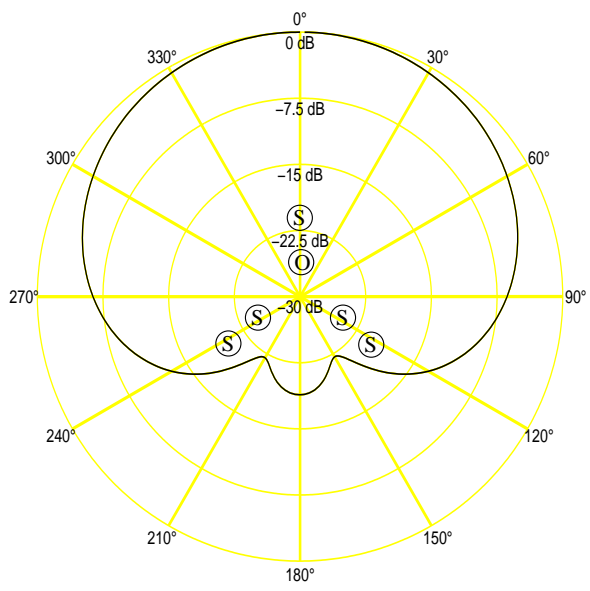

Figure 4: Power radiation pattern of the seven element monopole antenna shown in Figure 2 with two parasitics shorted $(S)$ to ground and one open $(O)$ plus three hardwired to ground $(S)$. 
The correlation coefficients for the complex voltage patterns corresponding to the power radiation patterns in Figure 3 and 4 are calculated assuming the Clark scenario [11] with pdf $S(\theta, \phi)=\delta(\theta-\pi / 2) /(2 \pi \sin \theta)$ to model a ring of dense sources on a horizon about the receiving antenna. We assume that three (two) parasitics are always shorted, to get four (three) different directions with $90^{\circ}\left(120^{\circ}\right)$ separation. This gives the correlation coefficient for adjacent patterns, or modes, for the parasitic antenna in Figure 3 as $\left|\rho_{i, i+1}\right|^{2}=$ 0.1157 and for opposite patterns $\left|\rho_{i, i+2}\right|^{2}=0.0120$. For the parasitic antenna in Figure 4 we get $\left|\rho_{i, i+1}\right|^{2}=0.1002$. The envelope correlation coefficients for the signals received from the modes are taken as $\rho_{e i j} \approx\left|\rho_{i j}\right|^{2}$ [12]. A well known rule of thumb is that "uncorrelated" signals in diversity branches corresponds to an envelope correlation lower than 0.5. Hence, the SPAs presented here would achieve a diversity gain in Clarke's scenario.

\subsection{Channel capacity}

The MIMO channel capacity is calculated using the SPA at the base station (BS) and/or at the user equipment (UE). We consider a single user, point to point communication over a flatfading channel with $N$ transmit modes (or array antenna elements) and $M$ receive modes (or array antenna elements) and no co-channel interference. The channel output corresponding to an input block spanning $T$ symbol times is

$$
\mathbf{Y}=\mathbf{H X}+\mathbf{V}
$$

where the received signal $\mathbf{Y}$ is $M \times T$, the fading channel $\mathbf{H}$ is $M \times N$, the codeword matrix $\mathbf{X}$ is $N \times T$ and the receiver noise $\mathbf{V}$ is a $M \times T$ matrix. The entries of the noise matrix are i.i.d. Complex Gaussian with zero mean. We assume that the channel is quasi-static, i.e. constant over the block of length $T$ symbols.

With $n$ parasitic elements, there are $2^{n}$ different modes, or settings of the switchable diodes. The transmit and receive modes of the parasitic antennas are chosen among these to minimize the envelope correlation. We assume that the receiver switches through and samples the chosen modes during one symbol interval. As the underlying concept here is angle diversity, we use a simple, yet detailed channel model that includes the spatial dimension. In [13], a simple model based on a circular disc of uniformly distributed scatterers placed around the UE. This model was extended to a MIMO scenario with general antenna radiation functions in [14]. Since the model is based on fundamental physics, the correlations between the different antenna arrangements, which are of great importance in this type of study, are physically motivated. Different channel scenarios can easily be obtained by varying the radius of the scattering disc as well as the location of the BS relative the disc [14].

The channel capacity of the following cases are investigated; BS antenna array-UE antenna array, BS antenna arrayUE SPA, BS SPA-UE SPA. When the array is used, omnidirectional antenna elements are assumed with $d_{B S}=2 \lambda$ spacing at the BS and $d_{U E}=0.5 \lambda$ spacing at the UE. Furthermore, we assume that the channel response is flat over frequency, an ap- proximation valid if the communication bandwidth $W$ is much less than the channel coherence bandwidth.

Shannon's capacity formula for a $N$ input, $M$ output MIMO channel, assuming equal power radiated from each transmitting antenna and $\mathbf{H}$ unknown at the transmitter can be written as

$$
C=\log _{2} \operatorname{det}\left[\mathbf{I}_{M}+\frac{\xi}{N} \mathbf{H H}^{H}\right]
$$

where $\xi / N$ is the signal to noise ratio (SNR) at each receive antenna. The matrix elements $\mathbf{H}_{i, j}$ represents the complex path gain from transmitter $j$ to receiver $i$.

Using the channel model [13, 14], the path gain can be written as

$$
\mathbf{H}_{i, j}=\sum_{l=1}^{L} \alpha_{l} \exp \left\{j k\left(r_{t_{j} \rightarrow s_{l}}+r_{s_{l} \rightarrow r_{i}}\right)\right\} g_{t_{j}}\left(\phi_{l}^{T}\right) g_{r_{i}}\left(\phi_{l}^{R}\right),
$$

where the number of scatterers are $L$ and $\alpha_{l}$ is the complex Gaussian distributed reflection coefficient with zero mean and unit variance. Furthermore, $r_{t_{j} \rightarrow s_{l}}$ and $r_{s_{l} \rightarrow r_{i}}$, denotes the distance from UE antenna $j$ to scatterer $j$ and scatterer $j$ to BS antenna $i$ respectively. Note that the wave number is denoted $k=2 \pi / \lambda$. Finally $g_{t_{j}}\left(\phi_{l}^{R}\right)$ and $g_{r_{i}}\left(\phi_{l}^{R}\right)$ are the complex voltage radiation patterns of the antennas. The channel matrix in (4) is normalized in the sense that $\|\mathbf{H}\|_{F}=\sqrt{M}$, where $F$ denotes the Frobenius norm.

As the channel matrix $\mathbf{H}$ is a function of the random position and reflection coefficients of the scatterers, the capacity $C$ in (4) is a random variable. With the SNR set to $4 \mathrm{~dB}$, a Monte Carlo simulation with 4000 trials was performed to calculate the Complementary Cumulative Distribution Function (CCDF) for a scenario with $L=20$ scatterers. The calculations are performed for an equal number of modes at transmit and receive $(N=M=4)$. The distance between the BS and UE is $D=50 \lambda$. The CCDF of the capacity is shown in Figure 5 for the scattering disc radius $R=50 \lambda$. The curves are compared to the channel matrix $\mathbf{H}$ with i.i.d. elements with a complex Gaussian distribution. Using the array antenna at the UE results in a slightly higher capacity than the array-parasitic configuration, however at the expense of more hardware due to the use of four transceivers instead of one. If the SPA is used at the BS, the capacity is further decreased, as the signals from the modes becomes correlated due to the small angular spread as seen at the BS.

The capacity at $10 \%$ outage is presented in Figure 6 and 7 for the two types of antenna configurations respectively. A large disc correspond to an indoor scenario, where both BS and UE are surrounded by scatterers. The other extreme, with a small scattering disc centered at the UE, as in an outdoor to indoor channel, results in a smaller capacity, due to the reduced angular spread, and hence, lower angle diversity gain. This can especially be seen in the case where the BS and the UE both are equipped with parasitic antennas, the capacity increases when the radius of the scattering disc exceeds the BS to UE distance, where full angular diversity also is possible at the BS. 


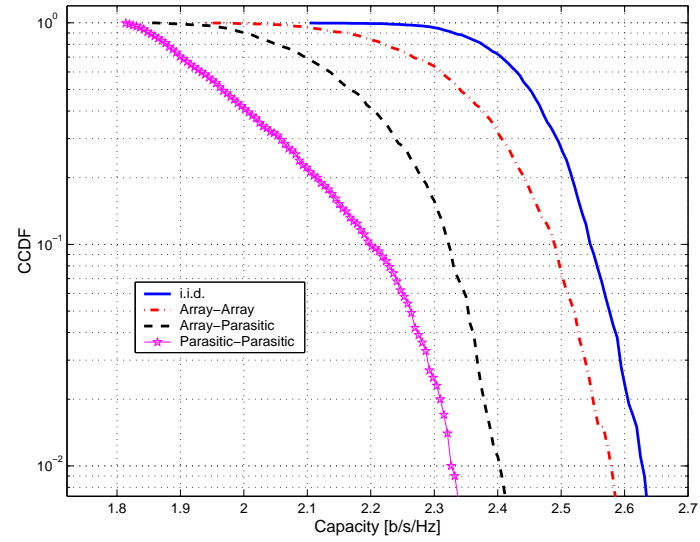

Figure 5: The complementary cumulative distribution function of the MIMO channel capacity for the $N=M=4$ case. The $\mathrm{SNR}$ is $4 \mathrm{~dB}$ and the scattering disc radius is $50 \lambda$. The parasitic antenna is shown in Figure 1.

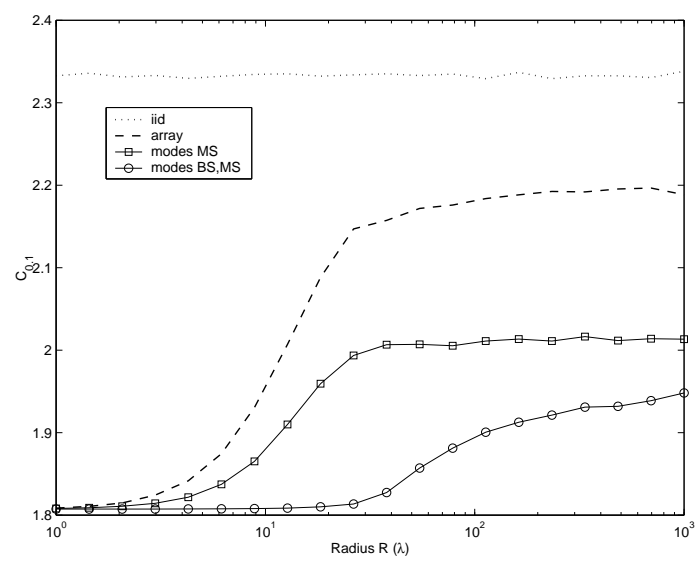

Figure 6: The channel capacity at $10 \%$ outage versus the radius of the scattering disc for the $N=M=4$ case. The parasitic antenna is shown in Figure 1. $\mathrm{SNR}=4 \mathrm{~dB}$.

At high bit rates, it might not be possible to switch through several modes during a symbol interval. Therefore a system with only two modes was investigated. The parasitic antenna in Figure 1 was used, but only two modes with lowest signal correlation (opposite in direction) was utilized. The capacity at $10 \%$ outage is shown in Figure 8. Comparing Figure 6 and 8 , we see that the overall capacity is lower, but the difference between the array and the SPA is slightly smaller at scattering disc radius around $10 \lambda$.

\section{EVALUATING BER USING STBC}

In this section we use space time block coding (STBC) [15] to exploit the available channel capacity discussed in the previous section. We assume that the transmitter have no knowledge of the channel state information and uses a very simple maximum likelihood detector based on linear processing at the receiver. The coding consists of mapping $P$ consecutive symbols $x_{1}, \ldots x_{P}$ onto the transmission matrix $\mathbf{X}$. The entries of $\mathbf{X}$ are linear combinations of $x_{1}, \ldots x_{P}$ and their conjugates.

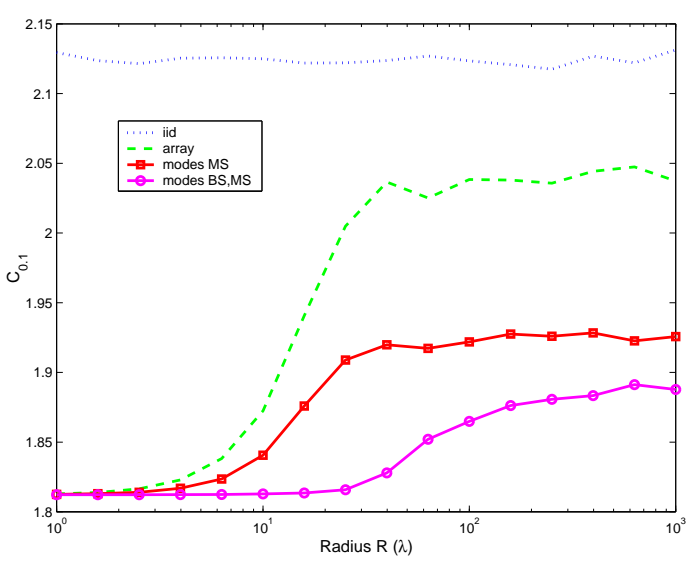

Figure 7: The channel capacity at $10 \%$ outage versus the radius of the scattering disc for the $N=M=3$ case. The parasitic antenna is shown in Figure 2.

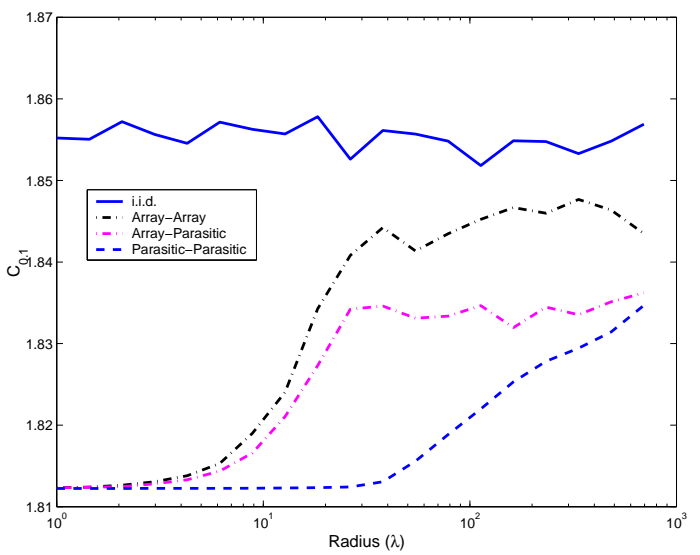

Figure 8: The channel capacity at $10 \%$ outage versus the radius of the scattering disc. Only $N=M=2$ of the 4 modes in the parasitic antenna in Figure 2 are used. $S N R=4 \mathrm{~dB}$.

For example, with $N=2$ transmit antennas we can use the Alamouti's scheme [16]

$$
\mathbf{X}=\left(\begin{array}{cc}
x_{1} & -x_{2}^{\star} \\
x_{2} & x_{1}^{\star}
\end{array}\right) .
$$

Since $P$ time slots are used to transmit $P$ symbols, the rate of the code is one. For complex symbol constellations, rate one STBC only exists for $N=2$ [15], however for real constellations there exists orthogonal, delay optimal rate one codes for $N=2,4,8$, as for example

$$
\mathbf{X}=\left(\begin{array}{cccc}
x_{1} & -x_{2} & -x_{3} & -x_{4} \\
x_{2} & x_{1} & x_{4} & -x_{3} \\
x_{3} & -x_{4} & x_{1} & x_{2} \\
x_{4} & x_{3} & -x_{2} & x_{1}
\end{array}\right)
$$

To study the performance in terms of BER using the SPA, we simulate the systems employing the STBCs (6) and (7), using the 4-direction SPA in Figure 3 and compare the results with the array antenna solution. When the Alamouti's STBC scheme is used, we use two opposite $\left(180^{\circ}\right.$ separation direction) modes as the two receiving/transmitting modes for the 
SPA. The BER for these configurations is compared in Figure 9. We use BPSK modulation in both cases, hence the bit rate $1 \mathrm{bit} / \mathrm{s} / \mathrm{Hz}$. It is observed that at the bit error rate of $10^{-2}$, the 2-mode SPA gives $8 \mathrm{~dB}$ gain over the uncoded system with one antenna and the 4-mode gives about $16 \mathrm{~dB}$ gain in SNR. The large and small scattering radius gives a difference in SNR gain less than $1 \mathrm{~dB}$. The array-array configuration is about 5 $\mathrm{dB}$ better than the array-SPA configuration.

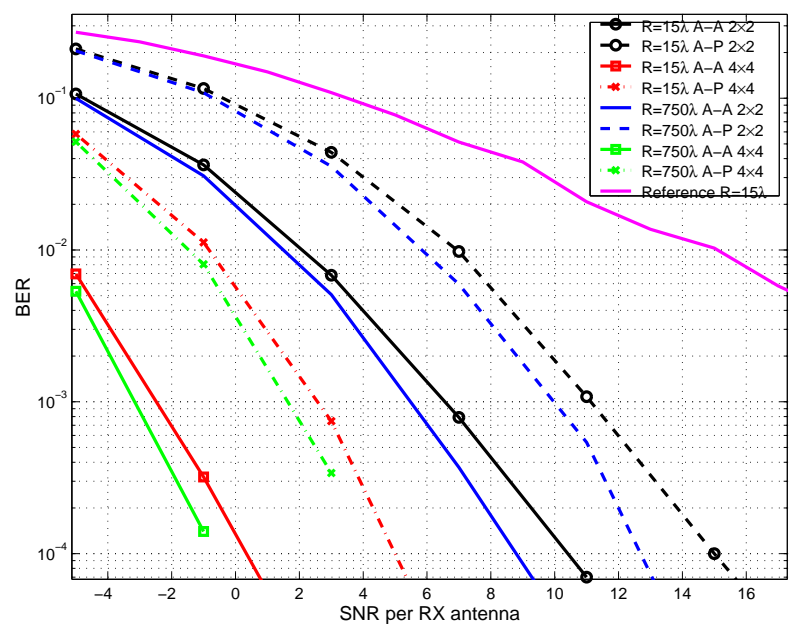

Figure 9: BER using STBC with $\mathrm{N}=\mathrm{M}=2$ and $\mathrm{N}=\mathrm{M}=4$ modes. The scattering disc radius is $750 \lambda$ and $15 \lambda$. The BER curves a compared to the $N=M=1$ reference antenna.

\section{CONCLUSIONS}

A MIMO system using a switched parasitic antenna has been analyzed, in terms of capacity and BER, assuming orthogonal space time block codes. The channel capacity was simulated using a spatial channel model. It was found that the SPA offered capacities close to the capacities offered by an array antenna, in realistic MIMO scattering environments. Since the SPA only requires one receiver, it could be an attractive low cost solution to future user terminals using space time coding to increase data rates. The examples of SPA:s presented here are only for demonstrative purposes, the design of the SPA depends on the dimensions of the user equipment.

\section{REFERENCES}

[1] G.J. Foschini and M.J.Gans. On limits of wireless communications in a fading environment when using multiple antennas. Wireless Personal Communications, 6:311-335, March 1998.

[2] A.A.Almhdie, V.Kezys, and T.D.Todd. Improved capacity in TDMA/SDMA using switched parasitic antennas. In Proceedings of IEEE Personal, Indoor and Mobile Radio Communications, pages 363-367, London,UK, 2000.

[3] N.L.Scott, O.L-Taylor, and R.G.Vaughan. Diversity gain from a single-port adaptive antenna using switched par- asitic elements illustrated with a wire and monolopole prototype. IEEE Transactions on Antennas and Propagation, 47:1066-1070, 1999.

[4] R.Vaughan. Switched parasitic elements for antenna diversity. IEEE Transactions on Antennas and Propagation, 47:399-405, 1999.

[5] S.L.Preston, D.V.Thiel, T.A.Smith, S.G.O'Keefe, and J.W.Liu. Base-station tracking in mobile communications using a switched parasitic antenna array. IEEE Transactions on Antennas and Propagation, 46:841844, 1998.

[6] D-S.Shiu, G.J.Foschini, M.J.Gans, and J.M.Kahn. Fading correlation and its effect on the capacity of multielement antenna systems. IEEE Transactions on Communications, 48(3):502-513, 2000.

[7] C.A. Balanis. Antenna Theory. John Wiley and Sons, Singapore, 1982.

[8] R.Schlub, D.V.Thiel, J.W.Lu, and S.G.O'Keefe. Dualband six-element switched parasitic array for smart antenna cellular communications systems. Electronic Letters, 36:1342-1343, 2000.

[9] S.L.Preston, D.V.Thiel, J.W.Lu, S.G.O'Keefe, and T.S.Bird. Electronic beam steering using switched parasitic patch elements. Electronic letters, 33:7-8, 1997.

[10] R.Vaughan and J.B.Andersen. Antenna diversity in mobile communications. IEEE Transactions on Vehicular Technology, 36:149-172, 1987.

[11] R.H.Clarke. A statistical theory of mobile radio reception. Technical report, Bell System Technical Journal, 1969.

[12] J.N. Pierce and S. Stein. "Multiple Diversity with Nonindependent Fading". Proc. of the IRE, 48:89-104, January 1960.

[13] P.Petrus, J.H.Reed, and T.S.Rappaport. Geometrically based statistical channel model for macrocellular mobile environments. In Proc. IEEE Global Telecommunications Conference, pages 1197-1201, 1996.

[14] T. Svantesson. An antenna solution for MIMO channels: The multimode antenna. In Proc. 34th Asilomar Conf. Sig., Syst., Comput., Pacific Grove, CA, October 2000.

[15] V.Tarokh, H.Jafarkhani, and A.R.Calderbank. Spacetime block codes from orthogonal design. IEEE Transactions on Communications, 45:1456-1467, 1999.

[16] S.M. Alamouti. A simple transmit diversity technique for wireless communications. IEEE Journal on selected areas in communications, 16:1451-1458, 1998. 